\title{
Initial Attitude Determination Using a Single Star Sensor
}

\author{
By Jinchun WANG, ${ }^{1,2)}$ Sangwoo CHO ${ }^{2)}$ and Joohwan CHUN ${ }^{1)}$ \\ ${ }^{1)}$ Scientific Computing Laboratory, Korea Advanced Institute of Science and Technology, South Korea \\ ${ }^{2)}$ National Security Research Institute, South Korea
}

(Received February 22nd, 2006)

\begin{abstract}
An initial quaternion estimation method for the attitude determination of a spacecraft using an onboard star sensor is presented. In this method, we use a sequence of the number of stars in the field of view (FOV) of the star sensor as the measurement instead of the direction vector pairs of stars. A new statistical observation model is derived and coupled with the kinematics model of attitude to develop a cost function of the estimated initial quaternion. The attitude acquisition method proposed herein exploits generalized simulated annealing to optimize the cost function and find the initial quaternion. In addition, a virtual sub-FOV and its shuffling procedure for a more accurate estimation are presented. The performance of the proposed method is quantified using an extensive simulation.
\end{abstract}

Key Words: Attitude Determination, Star Sensor, Simulated Annealing

\section{Introduction}

Over the past few decades, specialized acquisition maneuvers have been contrived for attitude acquisition capable of determining the initial orientation between a body coordinate system and the reference coordinate system. Wahba formulated an attitude estimation as a least-squares problem to obtain the attitude matrix from noisy measurement. ${ }^{1)}$ Various approaches, which solve the Wahba problem using a direct method or a filtering method, have been reported in literature..$^{2-5)}$ There are various attitude representation methods. ${ }^{6)}$ As some examples, the Direction Cosine Matrix (DCM) ${ }^{7)}$ the Euler angle, ${ }^{8)}$ the quaternion, ${ }^{9)}$ and the Rodrigues parameters ${ }^{10)}$ are used to express the attitude. Although the attitude representation method and the estimation scheme are different, the attitude is evaluated with the observation vectors in all of the above-mentioned methods. We can obtain the vector observations using a single sensor such as a star sensor, a sun sensor or a magnetometer, or a combination of these sensors. Although the fusion of multi-sensor data can help to increase the accuracy of estimation, the cost and complexity of the system also increases.

With the recent advancement of imaging devices, the method of attitude determination using a star sensor has been widely used. ${ }^{11)}$ In a star tracking system, the attitude is evaluated with star vector pairs, which are observed in the body frame and unit vector pairs of corresponding stars in the reference frame, which can be obtained from a star catalog by identifying the stars through a pattern-recognition method. Besides needing high computational power for the pattern-recognition process, ${ }^{12)}$ these methods usually require a preprocessing system using auxiliary sensors to determine the initial attitude. Though attitude acquisition methods using dual sensors achieve some level of the initial probability mass function (pmf) of the orientation, these

C 2007 The Japan Society for Aeronautical and Space Sciences methods do not provide a pmf capable of finding the optimal initial attitude.

Under the assumption that the three-axis angular velocity of the body can be precisely measured by onboard gyros, we present a new attitude acquisition method for onboard application of a star tracker, which uses a star sensor and provides more accurate initial attitude information than preprocessing sensors. We proposed a novel observation model for attitude determination using star sensors, which is not based on the direction vector of stars but uses discrete star density (i.e., the number of stars inside the field of view (FOV) of the star sensor). ${ }^{13)}$ In this work, we derive a more sophisticated observation model and apply this to the initial attitude acquisition problem. The proposed method estimates the attitude by optimizing a cost function, which compares the measurements with a reference star density obtained from a star catalog. Generalized simulated annealing (GSA) is exploited for the optimization procedure. In addition, a virtual sub-FOV and its shuffling procedure for a more accurate estimation are presented.

The preliminaries such as coordinate systems, attitude representation, and related kinematics equations are stated in Section 2. Then a statistical model of the measurement and an optimization solution to the problem are proposed in Section 3. A modified solution for more accurate estimations is also presented for both static and dynamic cases in Section 3. Evaluations of the proposed algorithm through computer simulations are given in Section 4, and concluding remarks are given in Section 5.

\section{Problem Statement}

\subsection{Attitude representation and propagation}

The Earth-centered inertial (ECI) coordinate system $\left(x_{\mathrm{e}}, y_{\mathrm{e}}, z_{\mathrm{e}}\right)$ with its origin at the center of the Earth is chosen as the reference coordinate system since it does not move 
with respect to stars. A coordinate system closely related to the ECI coordinate system is the celestial coordinate system, in which the location of an object projected against the celestial sphere is described by two angles called right ascension (RA) $\alpha$ and declination (Dec) $\beta$. The body-fixed (BF) coordinate system $\left(x_{\mathrm{b}}, y_{\mathrm{b}}, z_{\mathrm{b}}\right)$ is a coordinate system having its origin at the spacecraft's center of mass.

A quaternion spans the four-dimensional space with a basis $\{1, \boldsymbol{i}, \boldsymbol{j}, \boldsymbol{k}\}$. A quaternion is defined as $\boldsymbol{q}=q_{0}+q_{1} \boldsymbol{i}+$ $q_{2} \boldsymbol{j}+q_{3} \boldsymbol{k}$, by an ordered pair of a real number and a three-dimensional vector, $\boldsymbol{q}=\left(q_{0}, \boldsymbol{v}\right) \in \mathbb{R} \times \mathbb{R}^{3}$, where $\boldsymbol{v}=$ $\left[\begin{array}{lll}q_{1} & q_{2} & q_{3}\end{array}\right]^{\mathrm{T}}$. A quaternion of unit length is called a unit quaternion, which can be considered a point on the unit hyper-sphere $\mathbb{S}^{3}$. The orientation of a rigid body can be represented as a rotation about a fixed axis $\hat{\boldsymbol{v}}$ by an angle $\theta \in[0,2 \pi)$, where $\hat{\boldsymbol{v}}$ is a unit of $\boldsymbol{v}$. Any rotation matrix $R_{\boldsymbol{q}}$, which is equivalent to the quaternion $\boldsymbol{q}$, can be described as follows:

$$
R_{q}=\left[\begin{array}{ccc}
1-2\left(q_{2}^{2}+q_{3}^{2}\right) & 2\left(q_{1} q_{2}-q_{3} q_{0}\right) & 2\left(q_{3} q_{1}+q_{2} q_{0}\right) \\
2\left(q_{3} q_{0}+q_{1} q_{2}\right) & 1-2\left(q_{3}^{2}+q_{1}^{2}\right) & 2\left(q_{2} q_{3}-q_{0} q_{1}\right) \\
2\left(q_{3} q_{1}-q_{2} q_{0}\right) & 2\left(q_{2} q_{3}+q_{0} q_{1}\right) & 1-2\left(q_{1}^{2}+q_{2}^{2}\right)
\end{array}\right] .
$$

The rotation matrix is equivalent to the transpose of a DCM. If we know the exact angular velocity $\boldsymbol{w}=\left[\begin{array}{lll}\omega_{x} & \omega_{y} & \omega_{z}\end{array}\right]^{\mathrm{T}}$ for the entire time, the differential equations for the quaternion elements are given in the form

$$
\begin{gathered}
q_{0}=-\frac{1}{2}\left(\boldsymbol{v}^{\mathrm{T}} \boldsymbol{w}\right), \\
\dot{\boldsymbol{v}}=\frac{1}{2}\left(q_{0} \boldsymbol{w}+\boldsymbol{v} \times \boldsymbol{w}\right) .
\end{gathered}
$$

These are linear differential equations that can be integrated easily, given the initial condition (i.e., initial quaternion $\boldsymbol{q}_{t_{0}}$ and the forcing function $\boldsymbol{w}(t)$ ). In strapdown (analytic) navigation systems, once $\boldsymbol{q}$ is obtained, the rotation matrix is immediately given using Eq. (1).

Let $\boldsymbol{p}^{\mathrm{b}}=\left[\begin{array}{lll}x_{\mathrm{b}} & y_{\mathrm{b}} & z_{\mathrm{b}}\end{array}\right]^{\mathrm{T}} \in \mathbb{R}^{3}$ be the unit vector in the $\mathrm{BF}$ coordinate system. The corresponding vector $p^{\mathrm{e}}$ in the ECI coordinate system is obtained by multiplying the rotation matrix;

$$
\boldsymbol{p}^{\mathrm{e}}(t)=R_{\boldsymbol{q}_{t}} \cdot \boldsymbol{p}^{\mathrm{b}}(t) .
$$

If a spacecraft (or rigid body) has rotational motion, $\boldsymbol{p}^{\mathrm{e}}(t)$ is a time-varying quantity, because the quaternion is propagated by Eqs. (2) and (3). If we have an exact profile of $\boldsymbol{p}^{\mathrm{b}}(t)$, angular velocity $\boldsymbol{w}_{t_{0}: t}$ for the time after initial time $t_{0}$, and the initial quaternion $\boldsymbol{q}_{t_{0}}, \boldsymbol{p}^{\mathrm{e}}(t)$ can be computed in terms of $\boldsymbol{q}_{t_{0}}$ by the numerical integration of Eqs. (2) and (3):

$$
\boldsymbol{p}^{\mathrm{e}}(t)=R_{g\left(t ; \boldsymbol{q}_{t_{0}}, \boldsymbol{w}_{t_{0}: t}\right)} \cdot \boldsymbol{p}^{\mathrm{b}}(t)
$$

where $g(\cdot) \in \mathbb{S}^{3}$ is a nonlinear vector function that includes the numerical integration of Eq. (3). Consequently, once we know the angular velocity, the initial quaternion, and the unit vector profile in the BF coordinate system, which is the trajectory of the star sensor's view position, all the unit vectors that point out the focal direction of the star sensor in the ECI coordinate system can be reformulated in terms of the initial quaternion.

\subsection{Problem statement}

It is necessary to determine the orientation of the $\mathrm{BF}$ coordinate system, denoted by $\mathcal{V}$, relative to the ECI coordinate system, denoted by $\mathcal{U}$, from the star sensor images observed in the BF coordinate system and the reference map obtained from the star catalog. The goal of this paper is to compute $\hat{\boldsymbol{q}}_{t_{0}}$, which is the estimate of $\boldsymbol{q}$ at the initial time $t_{0}$ given the rotation matrix $R_{\boldsymbol{q}_{t_{0}}}$ based on noisy measurements.

\section{Initial Attitude Estimation}

The measurements are obtained from a star sensor with time intervals, and the number of stars in the FOV is used as a measurement. The measurement model can be obtained from a star catalog. Since the number of stars depends on the view position of the star sensor in the celestial sphere, we can estimate the attitude of the BF frame mounting the star sensor by comparing the measurements and the measurement model.

\subsection{Measurement model}

In this section, we derive the statistical measurement model of the number of stars detected in the FOV of a star sensor.

To obtain the statistical response of a star sensor for the number of stars detected inside its FOV from a star catalog, the following assumptions are required: First, all of the stars detected by the star sensor within the detection threshold are listed in the star catalog. This assumption is not always reasonable when we use an on board star catalog, which usually omits double stars and variable stars. However, since a star catalog including all stars brighter than the detection threshold are available and will be used on the ground in our algorithm, this assumption is a plausible one. Second, we have well-defined instrumental magnitudes for all of the stars; that is, the mean and standard deviations of the magnitude are given for each star. Because the standard deviation of magnitude of a star catalog is mainly affected by the sensor's optical characteristics, it is assumed that all stars have an identical standard deviation. Third, the star sensor has an exact detection threshold represented in the magnitude. Fourth, the resolution of the star sensor is sufficient to separate adjacent stars. Finally, the FOV of the star sensor has a circular shape so that the rotation of the body does not affect the measurement value.

Now, we derive the statistical response of the star sensor 
to count the number of stars in the FOV. Let $N_{\mathrm{p}}$ be the total number of stars in the FOV when the direction of the optical axis of the star sensor points to location $\boldsymbol{x}$, and let $m_{\mathrm{th}}$ be the magnitude threshold of the star sensor for the detection of stars. The magnitude of the $i_{\text {th }}$ star is normally distributed, $\mathcal{N}\left(m_{\mathrm{i}}, \sigma^{2}\right)$, from the second assumption. Therefore, the detection probability of the $i_{\text {th }}$ star is given by

$$
\begin{aligned}
p_{\mathrm{i}} & =P\left[m_{\mathrm{i}} \leq m_{\mathrm{th}}\right] \\
& =\int_{-\infty}^{m_{\mathrm{th}}} \frac{1}{\sqrt{2 \pi} \sigma} \exp \left(-\frac{\left(x-m_{\mathrm{i}}\right)^{2}}{2 \sigma^{2}}\right) \mathrm{d} x .
\end{aligned}
$$

Here, the inequality sign and the range of integral are represented in this way because the brighter the star is, the lower magnitude it has. Let $s_{\mathrm{i}} \in\{0,1\}$ be a random variate for the $i_{\text {th }}$ star of $N_{\mathrm{p}}$, where $s_{\mathrm{i}}=1$ means that the $i_{\text {th }}$ star is detected by the star sensor. Using Eq. (6), the mean $\mu_{\mathrm{i}}$ and variance $\sigma_{\mathrm{i}}^{2}$ of $s_{\mathrm{i}}$ are given by:

$$
\mu_{\mathrm{i}}=p_{\mathrm{i}}
$$

and

$$
\sigma_{\mathrm{i}}^{2}=p_{\mathrm{i}}\left(1-p_{\mathrm{i}}\right) .
$$

Let $\left(s_{1}, s_{2}, \ldots, s_{N_{\mathrm{p}}}\right)$ be a set of $N_{\mathrm{p}}$ independent random variates, and each $s_{\mathrm{i}}$ has a binomial distribution with a mean and finite variance given in Eqs. (7) and (8), respectively. Let $n_{\mathrm{p}}$ be defined as $n_{\mathrm{p}}=s_{1}+s_{2}+\cdots+s_{N_{\mathrm{p}}}$. If $N_{\mathrm{p}}$ is a number sufficiently large enough to apply the Central Limit Theorem, then the random variate $n_{\mathrm{p}}$ is approximately normally distributed with:

$$
\begin{gathered}
\mu_{n_{\mathrm{p}}}=\sum_{i=1}^{N_{\mathrm{p}}} p_{i}, \\
\sigma_{n_{\mathrm{p}}}{ }^{2}=\sum_{i=1}^{N_{\mathrm{p}}} p_{i}\left(1-p_{i}\right) .
\end{gathered}
$$

Consequently, when $N_{\mathrm{p}}$ stars with different means and the same variance exist inside the FOV for a given orientation of the star sensor, the number of stars detected by the star sensor has a normal distribution. Note that the mean and variance in Eqs. (9) and (10) are functions of the magnitude threshold, $m_{\mathrm{th}}$, and the direction of the sensor's optical axis (i.e., RA and Dec).

A star-density map is a two-dimensional map that has linear grids with respect to RA and Dec, and the value at the grid point is the mean value according to Eq. (9) for the star-density mean map. Since the density of the cloud depends on RA and Dec, the star-density map also depends on the location and the threshold of the magnitude of the star sensor. The quaternion $\boldsymbol{q}$ maps the vector of the sensor's optical direction $\boldsymbol{p}^{\mathrm{b}}$ into RA and Dec in the ECI coordinate, $\boldsymbol{x}(\boldsymbol{q})=\left[\begin{array}{ll}\alpha \beta & \beta\end{array}\right]^{\mathrm{T}}: \mathbb{S}^{3} \rightarrow \mathbb{R}^{2}$. Therefore, for a given $\boldsymbol{p}^{b}$, the reference star-density map is subject to the quaternion, and the threshold of the magnitude of the star sensor: $h\left(\boldsymbol{x}, m_{\mathrm{th}}\right)$.

Assuming that whole stars contained in the Smithsonian Astrophysical Observatory (SAO) star catalog are considered, and $m_{\mathrm{th}}=6.0$ of the star sensor with $30^{\circ} \mathrm{FOV}$, the star-density mean map, $h_{\mu}(\boldsymbol{x})$, is shown in Fig. 1. Here,

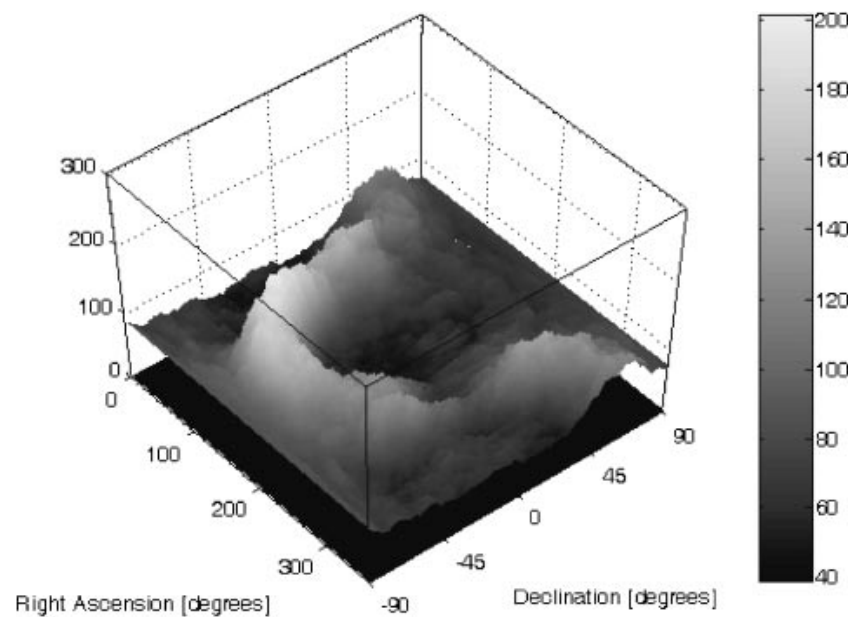

Fig. 1. Star-density mean map for $m_{\mathrm{th}}=6.0$.

the $m_{\mathrm{th}}$ is omitted because there is no ambiguity. The grid size of each star-density map is $1^{\circ} \times 1^{\circ}$. When $\boldsymbol{x}(\boldsymbol{q})$ is not located on the grid point, $h_{\mu}(\boldsymbol{x}(\boldsymbol{q}))$ is computed using bilinear interpolation as:

$$
\begin{aligned}
\hat{h}\left(\alpha_{0}, \beta_{0}\right)= & h\left(\alpha_{1}, \beta_{1}\right)+\left\{h\left(\alpha_{2}, \beta_{1}\right)-h\left(\alpha_{1}, \beta_{1}\right)\right\}\left(\alpha_{0}-\alpha_{1}\right) \\
& +\left\{h\left(\alpha_{1}, \beta_{2}\right)-h\left(\alpha_{1}, \beta_{1}\right)\right\}\left(\beta_{0}-\beta_{1}\right) \\
& +\left\{h\left(\alpha_{1}, \beta_{1}\right)+h\left(\alpha_{2}, \beta_{2}\right)-h\left(\alpha_{1}, \beta_{2}\right)\right. \\
& \left.-h\left(\alpha_{2}, \beta_{1}\right)\right\}\left(\alpha_{0}-\alpha_{1}\right)\left(\beta_{0}-\beta_{1}\right),
\end{aligned}
$$

where $\hat{h}\left(\alpha_{0}, \beta_{0}\right)$ is the interpolated value at position $\boldsymbol{x}(\boldsymbol{q})=\left[\begin{array}{ll}\alpha_{0} & \beta_{0}\end{array}\right]^{\mathrm{T}}$ and $\alpha_{i}$ and $\beta_{i}$ for $i=1,2$ are grid positions. Let $\eta$ be the digitization error. The error is caused by bilinear interpolation and a function of the state vector of a sample, $\eta(\boldsymbol{x}(\boldsymbol{q}))$. The digitization error has a uniform distribution where the mean and variance are dependent on the state vector of a sample and the four grid values of the star-density maps surrounding the sample. Therefore, the measured number of stars detected by the star sensor is modeled as:

$$
z_{\mathrm{m}}=\left\lceil\ell\left[h_{\mu}\left\{\boldsymbol{x}\left(\boldsymbol{g}\left(t ; \boldsymbol{q}_{t_{0}}, \boldsymbol{w}_{t_{0}: t}\right)\right)\right\}\right]+\eta\left[\boldsymbol{x}\left(\boldsymbol{g}\left(t ; \boldsymbol{q}_{t_{0}}, \boldsymbol{w}_{t_{0}: t}\right)\right)\right]\right\rceil,
$$

where $\lceil\cdot\rceil$ is a ceiling operator, which denotes the quantization error, and $\ell[\cdot]$ is the bilinear operator.

\subsection{Attitude estimation method}

The cost function $J\left(\boldsymbol{q}_{t_{0}}\right)$ is the sum of the absolute values of the difference between the measured number of stars, $z_{k}$, and measurement model in Eq. (12):

$$
\begin{aligned}
J\left(\boldsymbol{q}_{t_{0}}\right)= & \frac{1}{N} \sum_{k=1}^{N} \lambda\left(z_{k}\right) \mid z_{k}-\left\lceil\ell\left[h_{\mu}\left\{\boldsymbol{x}\left(\boldsymbol{g}\left(t_{k} ; \boldsymbol{q}_{t_{0}}, \boldsymbol{w}_{t_{0}: t_{k}}\right)\right)\right\}\right]\right. \\
& \left.\left.+\eta\left[\boldsymbol{x}\left(\boldsymbol{g}\left(t_{k} ; \boldsymbol{q}_{t_{0}}, \boldsymbol{w}_{t_{0}: t_{k}}\right)\right)\right]\right\rceil\right]
\end{aligned}
$$

where $N$ is the number of measurements, and $\lambda\left(z_{k}\right)$ is the normalizing factor given as $\lambda\left(z_{k}\right)=\exp \left(20 / z_{k}\right)$. This normalizing factor is chosen under consideration of the distribution of $z_{k}$ so that if $z_{k}$ is the more likely measurement, then the cost function has a lower value.

Figure 2 shows examples of the cost value with two varying quaternion parameters. In these examples, because we consider the unit quaternion, we cannot change only 


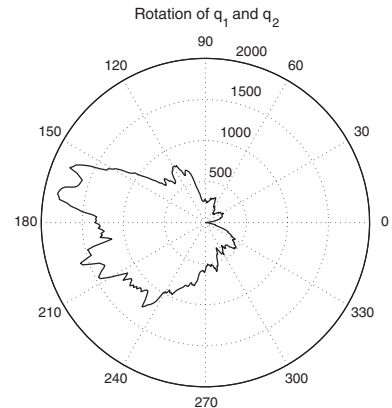

(a)

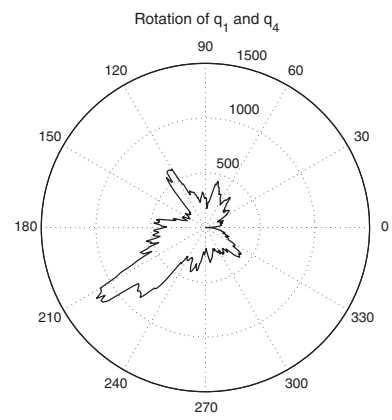

(c)

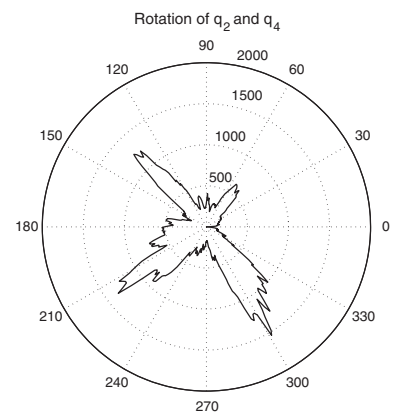

(e)

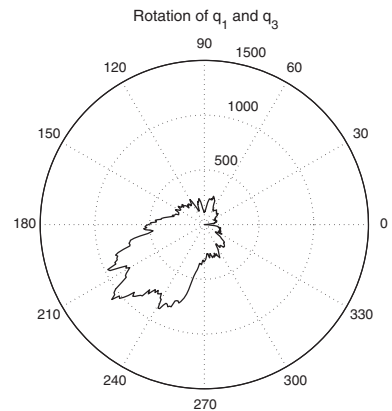

(b)

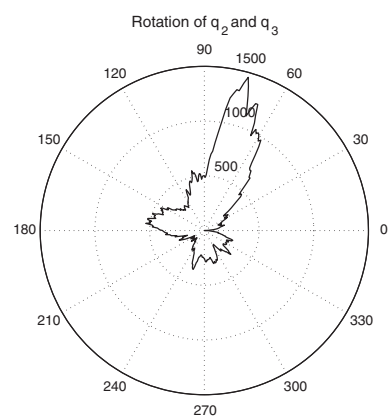

(d)

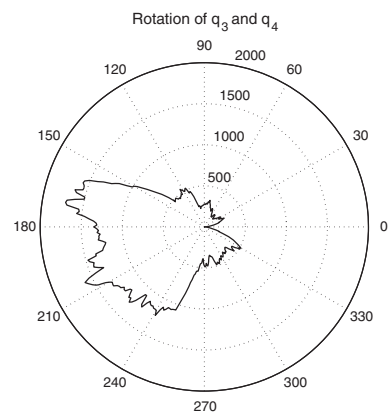

(f)
Fig. 2. Behavior of cost according to change in the angle between two quaternion parameters. (a) Rotation of $q_{1}$ and $q_{2}$. (b) Rotation of $q_{1}$ and $q_{3}$. (c) Rotation of $q_{1}$ and $q_{4}$. (d) Rotation of $q_{2}$ and $q_{3}$. (e) Rotation of $q_{2}$ and $q_{4}$. (f) Rotation of $q_{3}$ and $q_{4}$.

one parameter of the quaternion. Therefore, changing two parameters of the quaternion is defined by:

$$
\begin{array}{r}
{\left[\begin{array}{l}
q_{i}{ }^{\prime} \\
q_{j}{ }^{\prime}
\end{array}\right]=\left[\begin{array}{cc}
\cos \theta & \sin \theta \\
-\sin \theta & \cos \theta
\end{array}\right]\left[\begin{array}{l}
q_{i}^{\mathrm{t}} \\
q_{j}^{\mathrm{t}}
\end{array}\right]} \\
(i \neq j \text { and } i, j=0,1,2,3),
\end{array}
$$

where the superscript $\mathrm{t}$ represents the true value. The state (or search) space is $\left|q_{i}\right| \leq 1,(i=0,1,2,3)$ and $\|\boldsymbol{q}\|=1$. Note that there are several local minima in the cost functions, as shown in Fig. 2.

Our problem is to find a value of $\boldsymbol{q}_{t_{0}}$ that minimizes $J\left(\boldsymbol{q}_{t_{0}}\right)$ iteratively starting from an initial guess, $\hat{\boldsymbol{q}}_{t_{0}}$, to determine the initial attitude. However, the main difficulty of this approach is the presence of multiple local minima, as shown in Fig. 2. Using a descending-type optimization method such as the secant method or the downhill simplex method may find the local minimum. In this paper, we propose using the
Table 1. Modified generalized simulated annealing algorithm.

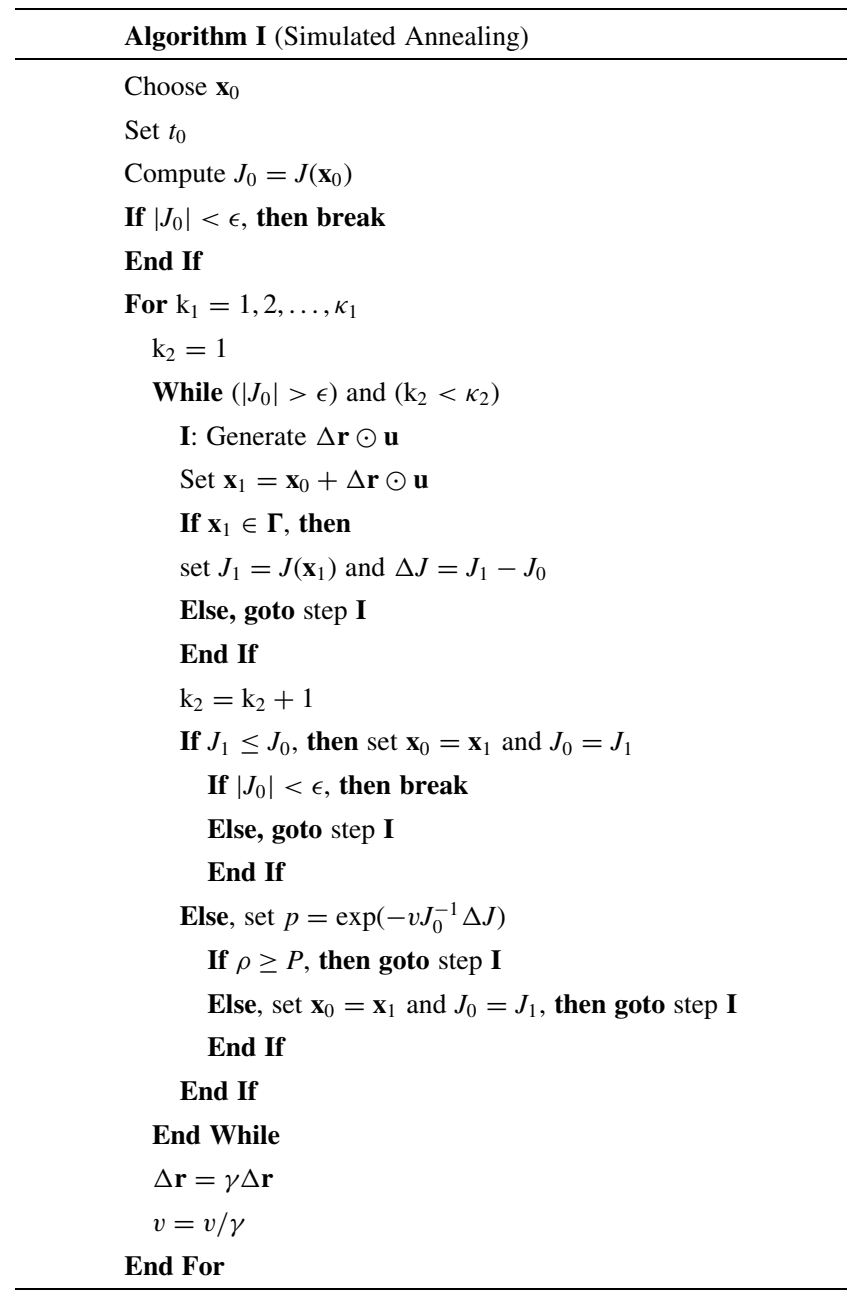

GSA global optimization method, which is an iterative optimization method for the nonlinear minimization problem. ${ }^{14)}$ The GSA technique, in our case, is summarized in Table 1. In Table 1, $\odot$ denotes the element-wise product.

\subsection{Shuffling of view positions}

The proposed algorithm estimates the initial quaternion by finding out the sensor's optical direction sequences. This process is performed by comparing the measurements to the measurement model (i.e., the star-density map). If the measurements sequence has a small variance, the resultant estimated quaternion may have large orientation errors.

To overcome this problem, we introduce a virtual subFOV and a shuffling method for the optical direction of this sub-FOV to make the variance between the measurements larger. A sub-FOV is a small FOV region inside the sensor's physical FOV, called the main-FOV; the direction vector to the center of a sub-FOV becomes a new unit vector in the BF coordinate system, as shown in Fig. 3. Since the star density is related to the view positions, we shuffle (i.e., randomly select in the main-FOV) a fictitious optical direction between the measurements so as to variate the positions of the sub-FOV and use the number of stars in the subFOV instead of that in the main-FOV. Figure 4 shows an example of the shuffling of the view positions. In Fig. 4, diamond markers represent the view position progress of 


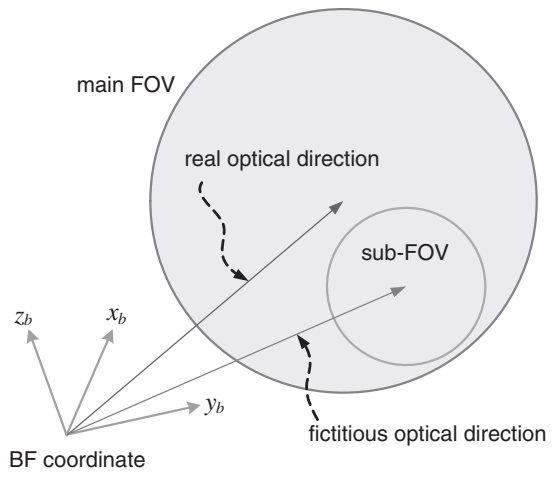

Fig. 3. Sub-FOV concept.

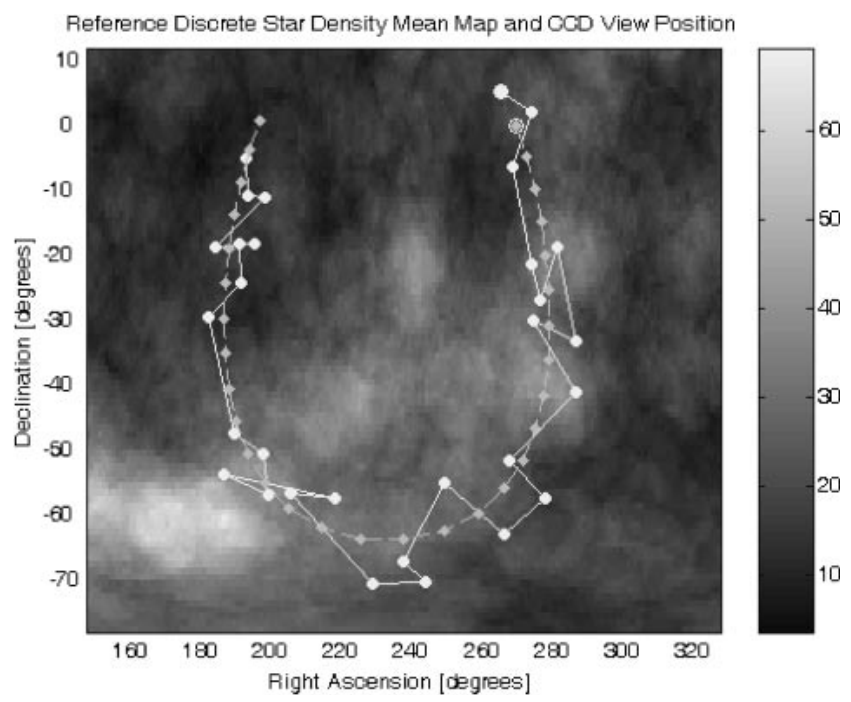

Fig. 4. Example of sub-FOV shuffling.

the focal direction of the star sensor, and circle markers indicate that of the direction vector of the sub-FOV. For the implementation of the sub-FOV method, we do not need the star-density map, which is generated with the main-FOV, but only the map with the sub-FOV.

The GSA technique can evade a convergence problem using a random step size at the cost of convergence time. There can be several points in the sky having the same number of stars in the FOV. However, this does not cause a misestimation since we can discriminate the right position by adopting a sub-FOV technique. The above two facts guarantee stable convergence of the proposed algorithm.

\section{Simulation Results}

Comprehensive simulation studies were made to investigate the acquisition of the initial attitude using computersimulated data. To evaluate the performance of the estimator, a convergence index, quoted from Bar-Itzhack and Idan, ${ }^{8)}$ is defined as:

$$
C \triangleq\left\|R_{\boldsymbol{q}_{t_{0}}}-R_{\hat{\boldsymbol{q}}_{t_{0}}}\right\|_{F}^{2},
$$

where $\|\cdot\|_{F}$ is the Frobenius norm of a matrix, $R_{\boldsymbol{q}_{t_{0}}}$ is the

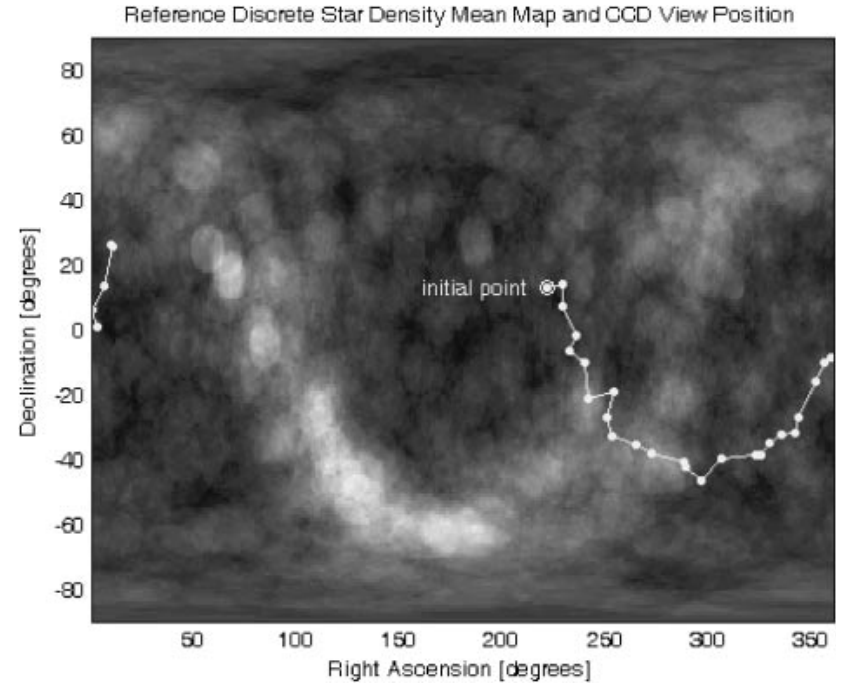

Fig. 5. Initial point of the direction vector and the sequential measurement points in the dynamic case.

rotation matrix of the true initial quaternion $\boldsymbol{q}_{t_{0}}$, and $R_{\hat{\boldsymbol{q}}_{t_{0}}}$ is that of the estimated initial quaternion $\hat{\boldsymbol{q}}_{t_{0}}$.

The performance of the proposed attitude estimator was evaluated for the following two cases: One is a static case, in which the body coordinate system $\mathcal{V}$ does not rotate relative to the reference system $U$, and the other is a dynamic case in which $\mathcal{V}$ rotates at an angular rate relative to $\mathcal{U}$. In our simulations, it is assumed that the star sensor has a main-FOV of $30^{\circ}$ and the sub-FOV is postulated to $15^{\circ}$. The magnitude threshold of detection stars is 6 . The annealing schedule used for both cases is composed of a control parameter $v=1.5$ with a reduction factor $\gamma=1 / \sqrt{2}$, and step size $\Delta \boldsymbol{r}=\left[\begin{array}{llll}r_{0} & r_{1} & r_{2} & r_{3}\end{array}\right]^{\mathrm{T}}$, where $r_{i}(i=0,1,2,3)$ is random numbers of the uniform distribution on $[-1,1]$, the control step $\kappa_{1}$ is 3000 , and the number of iterations $\kappa_{2}$ is 2000. The threshold is chosen as $\epsilon=5 \times 10^{-6}$. We start iterations with an initial state of randomly generated unit quaternions.

\subsection{Dynamic case}

In this simulation, the BF coordinate system $\mathcal{V}$ has a spinning motion at an angular velocity $\boldsymbol{w}=$ $\left[\begin{array}{lll}-0.1 & 0.2 & -0.1\end{array}\right]^{\mathrm{T}}(\mathrm{deg} / \mathrm{s})$. The time interval between measurements is 30 , and the number of measurements $N$ is 30 . Figure 5 shows the initial location of the direction vector and the sequential $N$ measurement positions on the stardensity map. The true initial quaternion and the estimated one are listed in Table 2. The convergence index given in Table 2 exhibits the estimation accuracy of the suggested method. The convergence behaviors of the quaternion's parameters are presented in Fig. 6. The estimate converges near the global minimum prior to the first control step.

\subsection{Static case}

Since there's no motion of the BF coordinate system in this case, we should obtain $N$ measurements by shuffling a fictitious sub-FOV inside the main-FOV. To avoid concentrations of the sub-FOV region around the sensor's optical direction, which results in a small variance in the measurements sequence, we forced the circle of the sub-FOV to be 
Table 2. True and estimated values.

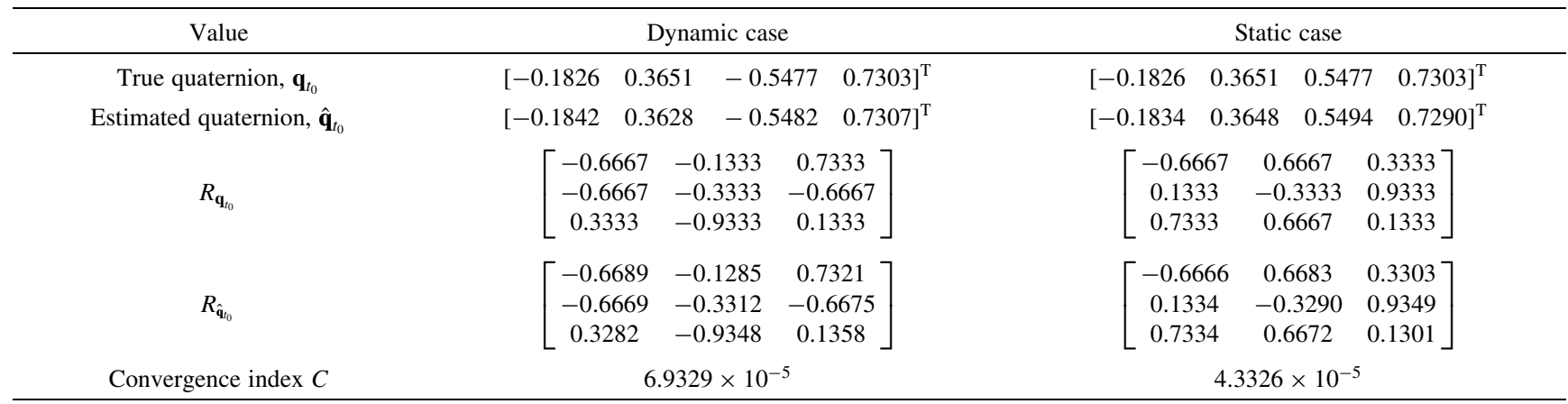
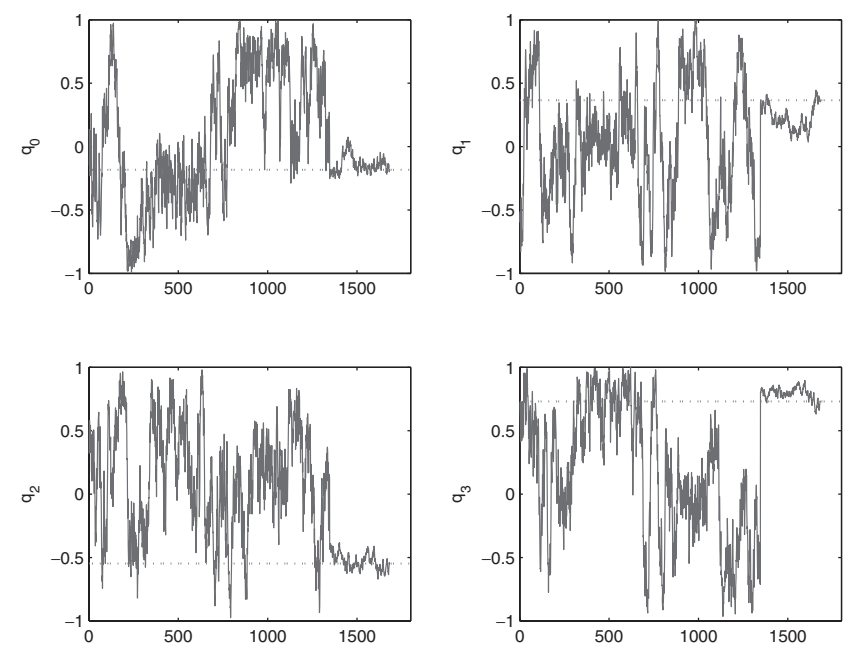

Fig. 6. Behaviors of quaternion parameters in the dynamic case. Dotted lines represent the true value of the parameter.

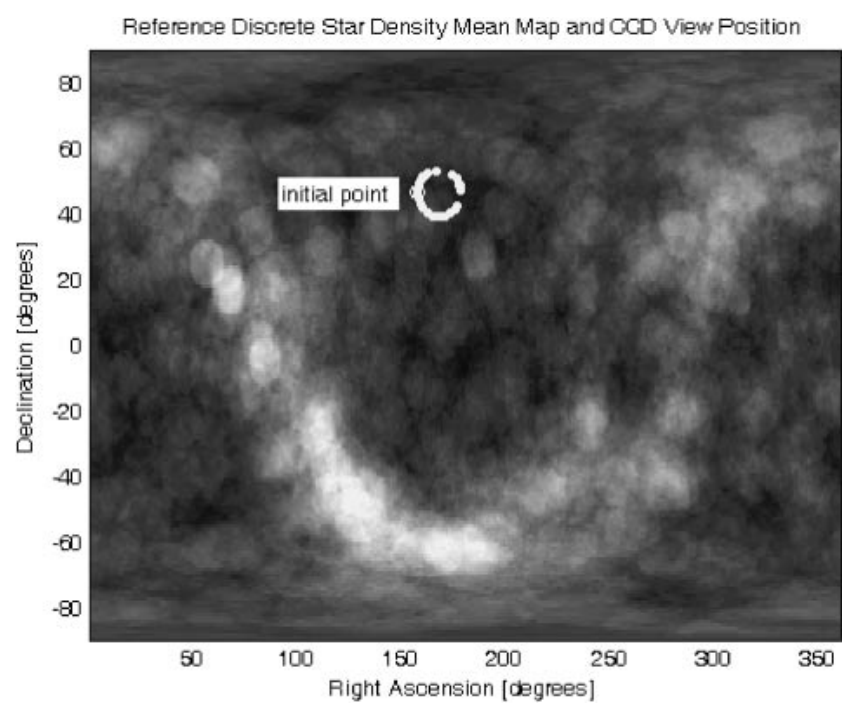

Fig. 7. Initial point of the direction vector and the sequential measurement points in the static case.

tangent inside that of the main-FOV. The resulting measurement locations are depicted in Fig. 7. Note that this measurement acquisition can be performed without a time delay between the measurements.

The view location of the optical direction in this example is deliberately selected in the small star density region to
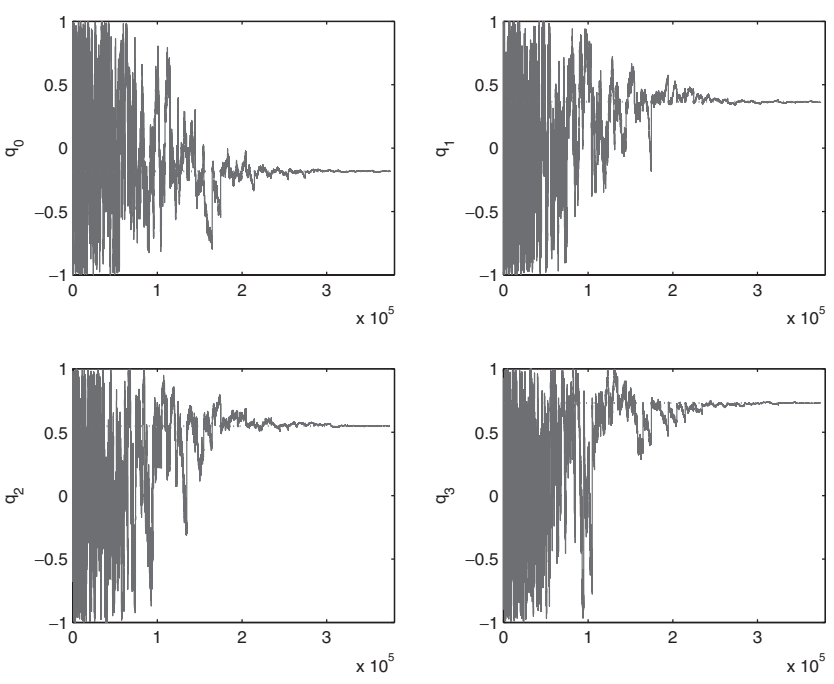

Fig. 8. Behaviors of quaternion parameters in the static case. Dotted lines represent the true value of the parameter.

show the acquisition ability of the proposed method even under unfavorable conditions. The results of the estimation of the static case are presented in Table 2. The corresponding results are plotted in Fig. 8, in which the convergence behavior of the quaternion's parameters are shown. In the static case, the iteration number for convergence is larger than that of the dynamic case because we use the same scheduling for comparison, and it can be reduced through more sophisticated scheduling tailored to the static case.

\section{Conclusions}

In this paper, we presented an initial quaternion estimation method for the attitude determination of a spacecraft based on a generalized simulated annealing technique using an onboard star sensor. Our method used the star sensor itself for determination of the initial attitude by using the number of stars in a FOV as the measurements. A new statistical observation model was presented, and a shuffling procedure for sub-FOVs, which resulted in more accurate estimates by enlarging the measurements' variance, was suggested. Attitude estimation algorithms were derived for the two cases: One for the static case, in which the orientation between the two coordinate systems is constant; and the second for the dynamic case, in which the BF coordinate 
system has an angular motion. Thus, our method can be used regardless of whether the spacecraft is spin-stabilized or three-axis-stabilized.

To verify the performance of the proposed method, computer simulations had been made by changing the rotational motion parameters. The simulation results demonstrated that the attitude can be estimated accurately.

Though it can be used for ground-based analysis, the proposed method is designed for onboard realtime application with star trackers. The GSA technique usually needs some convergence time, however, it is impossible to predict the exact convergence time because it is an iterative search method. In our simulation, the GSA method converges to the right attitude parameters in tens of thousands of iterative steps except for the worst cases, as in our static case simulation result, which required hundreds of thousands of iterations. Since the cost function needs only $O(N)$ computation, the realtime implementation of the proposed algorithm is practicable considering the computational capacities of DSPs available these days.

\section{References}

1) Wahba, G.: A Least Squares Estimate of Spacecraft Attitude, SIAM Rev., 7 (1965), p. 409.

2) Shuster, M. D. and Oh, S. D.: Three-Axis Attitude Determination from
Vector Observations, J. Guid. Control. Dynam., 4 (1980), pp. 70-77.

3) Lefferts, E. J., Markley, F. L. and Shuster, M. D.: Kalman Filtering for Spacecraft Attitude Estimation, J. Guid. Control Dynam., 5 (1982), pp. 417-429.

4) Markley, F. L.: Attitude Determination Using Vector Observations and the Singular Value Decomposition, J. Astronaut. Sci., 36 (1988), pp. 245-258.

5) Psiaki, M. L.: Attitude-Determination Filtering via Extended Quaternion Estimation, J. Guid. Control. Dynam., 23 (2000), pp. 206-214.

6) Shuster, M. D.: A Survey of Attitude Representations, J. Astronaut. Sci., 41 (1993), pp. 445-453.

7) Bar-Itzhack, I. Y. and Reiner, J.: Recursive Attitude Determination from Vector Observations: DCM Identification, J. Guid. Control Dynam., 7 (1984), pp. 51-56.

8) Bar-Itzhack, I. Y. and Idan, M.: Recursive Attitude Determination from Vector Observations: Euler Angle Estimation, J. Guid. Control Dynam., 10 (1986), pp. 152-157.

9) Bar-Itzhack, I. Y. and Oshman, Y.: Attitude Determination from Vector Observations: Quaternion Estimation, IEEE Trans. Aero. Elec. Sys., 21 (1985), pp. 128-135.

10) Idan, M.: Estimation of Rodrigues Paramters from Vector Observations, IEEE Trans. Aero. Elec. Sys., 32 (1996), pp. 578-586.

11) Liebe, C. C.: Star Trackers for Attitude Determination, IEEE Magz. Aero. Elec. Sys., 10 (1995), pp. 10-16.

12) Liebe, C. C.: Pattern Recognition of Star Constellations for Spacecraft Applications, IEEE Magz. Aero. Elec. Sys., 8 (1993), pp. 31-39.

13) Cho, S. and Chun, J.: Satellite Attitude Acquisition Using Dual Star Sensors with a Bootstrap Filter, Proc. IEEE Sensors 2002, Vol. 2, 2002, pp. 1723-1727.

14) Bohachevsky, O., Johnson, E. and Stein, L.: Generalized Simulated Annealing for Function Optimization, Technometrics, 28 (1986), pp. 209-217. 